Measur ement $s$ of $r$ ot at $i$ onal $t r$ ansf or m due to noni nductive toroi dal current usi ng moti onal Stark effect spectroscopy in the Large Hel i cal Devi ce

\begin{tabular}{|l|l|}
\hline $\begin{array}{l}\text { j our nal or } \\
\text { publ i cat i on t i t l e }\end{array}$ & Revi ew of Sci ent i f i c I nst r ument s \\
\hline vol une & Vol . 76 \\
\hline number & I ssue5 \\
\hline page $r$ ange & pp. 053505-1 - 053505- 5 \\
\hline year & 2005- 05- 01 \\
\hline URL & ht t p: //hdl . handl e. net /10655/119 \\
\hline
\end{tabular}




\title{
Measurements of rotational transform due to noninductive toroidal current using motional Stark effect spectroscopy in the Large Helical Device
}

\author{
K. Ida, ${ }^{\text {a) }}$ M. Yoshinuma, K. Y. Watanabe, T. Kobuchi, and K. Nagaoka \\ National Institute for Fusion Science, Toki 509-5292, Japan
}

(Received 14 October 2004; accepted 7 March 2005; published online 22 April 2005)

\begin{abstract}
The change of rotational transform due to noninductive plasma current driven by a negative neutral beam, which has a value typically less than $10 \%$ of total rotational transform determined by the external current in the helical coils, is measured in the Large Helical Device with motional Stark effect spectroscopy. Radial profiles of rotational transform are derived from the radial profiles of the polarization angle of the $\sigma$ component in the $H \alpha$ line emitted from the high energy hydrogen atom of the beam with four sets of linear polarizers, spectrometers, and CCD detectors. The radial profile of the change in the rotational transform due to the noninductive toroidal current driven by the neutral beam is measured. (C) 2005 American Institute of Physics. [DOI: 10.1063/1.1898943]
\end{abstract}

\section{INTRODUCTION}

The measurement of the rotational transform of the magnetic field, $\iota$, which is called $q=(1 / \iota)$ in tokamaks, has been recognized to be important in understanding the mechanism of transport improvement, since transport in plasma has been found to be sensitive to magnetic shear and/or rational surfaces (at rational $q$ values). ${ }^{1-3}$ In stellarators, the rotational transform is mainly determined by the vacuum magnetic field produced by external coils. The measurement of rotational transform of the magnetic field is considered to be important in stellarators as well as in tokamaks, because the characteristics of electron transport, especially in the internal transport barrier, ${ }^{4-6}$ and low- $n$ ideal MHD instability strongly depend on the rotational transform profiles. Therefore the measurements of rotational transform, $\iota$, are necessary even in the Large Helical Device, where more than $90 \%$ of the poloidal magnetic field at the plasma boundary is produced by external coils.

Motional Stark effect spectroscopy (MSE) has been a useful tool to measure the radial profile of the rotational transform. ${ }^{7-11}$ In nonaxisymmetric magnetic configurations, there is an intrinsic difficulty in the MSE measurements, because the pitch angle of the magnetic field changes in the toroidal direction (even changing its sign) along the line of sight within the beam width. The variation of the local pitch angle in the toroidal direction is even larger than that in the radial direction. Preliminary measurements of pitch angle in a Heliotron device using MSE spectroscopy were done in CHS. ${ }^{12}$ The diagnostic neutral beam with a narrow beam width was used to minimize the integration effect of the polarization angle in the region of varying pitch angle. Because of the low intensity of the diagnostic beam, the error bar of the measured pitch angle was too large to consider the small change in rotational transform due to non-inductive plasma current (typically less than 10\% of total equivalent plasma current). In the Large Helical Device, the change of rational

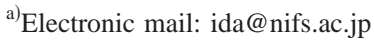

transform due to the noninductive plasma current driven by the tangentially injected neutral beam is typically on the order of 0.1 (order of $1^{\circ}$ in polarization angle) and requires a heating beam which has enough beam intensity to derive a precise measurement of pitch angle.

Therefore a technique is needed to minimize the integration effect of the polarization angle along the line of sight within a beamwidth, where the pitch angle of the magnetic field changes significantly. The integration effect results in a large variation of the polarization angle in wavelength within the $\sigma$ and $\pi$ components of the $H \alpha$ light, because the Doppler shift at the observation point (intersection of line of sight and each beamlet) slightly changes within the beamwidth. This variation of polarization angle in wavelength reaches $5^{\circ}$ to $10^{\circ}$ and it is much larger than the error bar required for the measurements. Although the pitch angle of the magnetic field produced by the external coils changes its sign along the toroidal direction, the magnetic field produced by the noninductive plasma current driven by the neutral beam has nearly toroidal symmetry near the plasma center. Therefore the variation of the polarization angle in wavelength due to the noninductive plasma current is much smaller than that due to the external coils (vacuum field). Therefore the integration effect can be eliminated by subtracting the polarization angles in the vacuum field from that measured at each wavelength. In this article, a MSE spectroscopy system is described which minimizes the integration effect and derives the rotational transform profiles precisely enough to consider the small changes from the vacuum magnetic field.

\section{EXPERIMENTAL SET UP OF MOTIONAL STARK EFFECT SPECTROSCOPY (MSE) IN LHD}

Figure 1 shows the experimental setup for MSE spectroscopy installed in the Large Helical Device. The LHD is a toroidal helical magnetic device (Heliotron device) with a major radius of $R_{\mathrm{ax}}=3.5-4.1 \mathrm{~m}$, an average minor radius of $0.6 \mathrm{~m}$, and a magnetic field $B$ of $0.5-3 \mathrm{~T}$. The major radius 


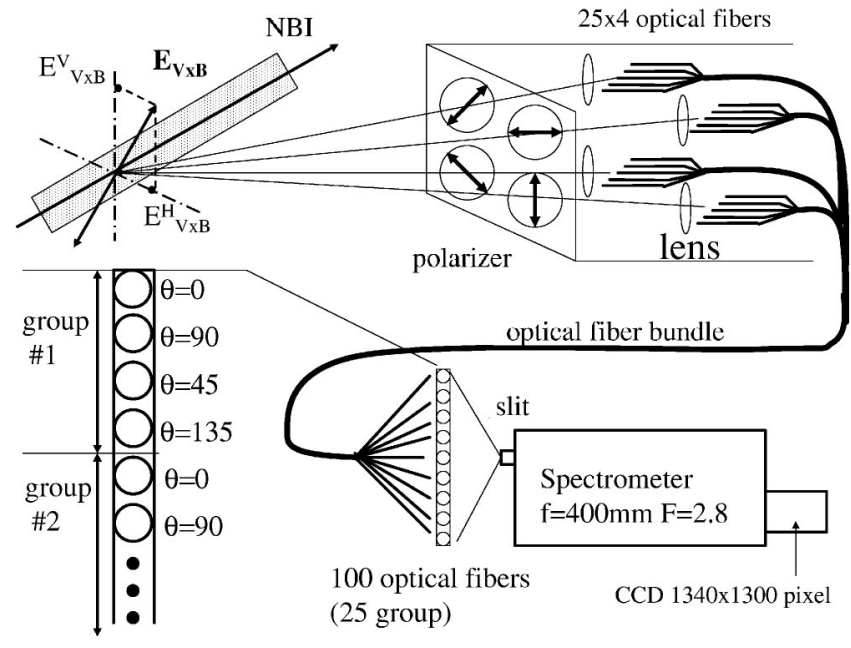

FIG. 1. Experimental setup for motional stark spectroscopy in LHD.

is $R_{\mathrm{ax}}=3.6 \mathrm{~m}$, the magnetic field $B$ is $1.5 \mathrm{~T}$, and the beam energy for MSE measurements is $146 \mathrm{keV} / \mathrm{amu}$ in this experiment. The plasma current equivalent to the edge poloidal magnetic field is $1.5 \mathrm{MA}$ in this experiment. The MSE system consists of a spectrometer, optical fibers, and linear polarizers tilted by $0^{\circ}, 45^{\circ}, 90^{\circ}$, and $135^{\circ}$ with respect to the horizontal direction. The linear polarizers are arranged in front of the object lenses to avoid a change of polarization angle due to the lenses. The 100 optical fibers with a core diameter of $200 \mu \mathrm{m}$ and $250 \mu \mathrm{m}$ of cladding are arranged at the focal plane of each object lens. The optical fibers are divided into 25 groups and each group has optical fibers with the linear polarizer tilted by $0,45,90$, and 135 with respect to the horizontal direction. The object lens of each optical fiber array is adjusted to make all the optical fibers in the same group to view an identical observation point (intersection between beam line and line of sight). The 100 optical fibers are arranged at the entrance slit of a high throughput spectrometer (Bunkou-Keiki CLP-400) which consists of two sets of $f=400 \mathrm{~mm} / F=2.8$ camera lenses and a $2160 / \mathrm{mm}$ grating. A backilluminated CCD $(1340 \times 1300$ with $20 \times 20 \mu \mathrm{m}$ pixel) at the exit plane is used to measure 100 spectra.

Figure 2 shows the CCD image for the $H \alpha$ emission

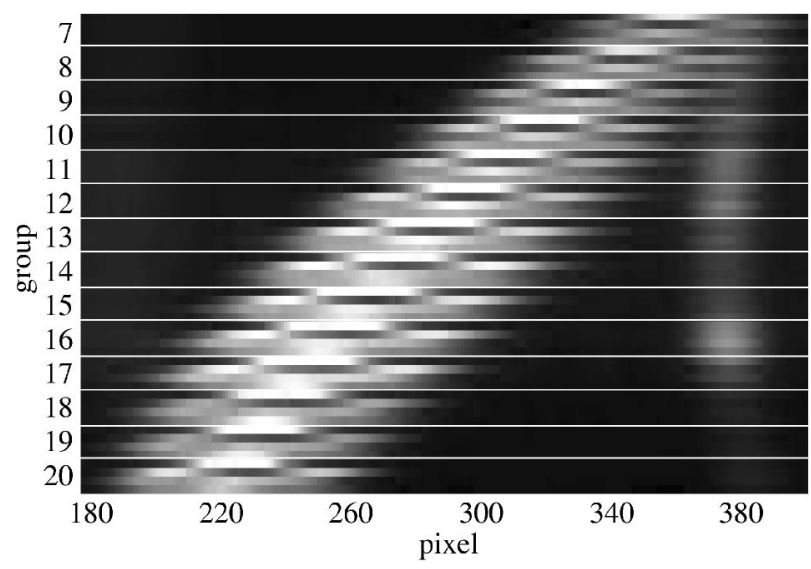

FIG. 2. CCD image for the spectra of Doppler shifted $H \alpha$ emission from beam hydrogen atoms.
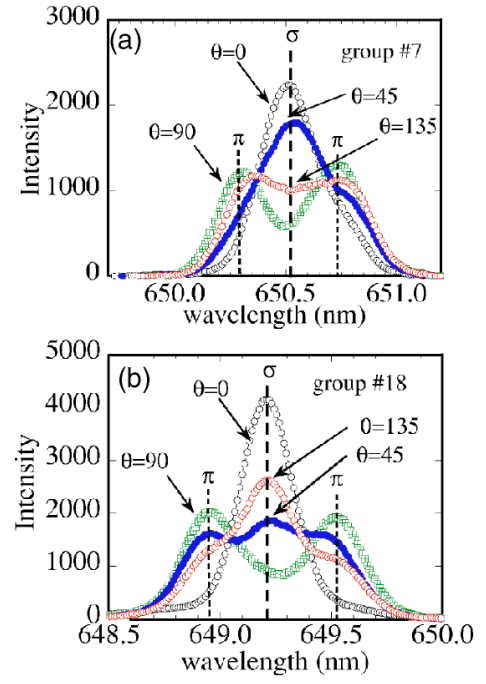

FIG. 3. (Color online) Spectra of Doppler shifted $H \alpha$ emission for (a) group 7 and (b) group 18 in Fig. 2.

emitted from the neutral beam measured with different polarization angles. Each group has four spectra with $0^{\circ}, 45^{\circ}$, $90^{\circ}$, and $135^{\circ}$, linear polarizers starting from the top. Clear peaks corresponding to the $\sigma$ components (which are parallel to the magnetic field) are observed for the spectra with a $0^{\circ}$ linear polarizer at the top of each group. Two peaks with a separation of 40-50 pixel are observed for the spectra with the $90^{\circ}$ linear polarizer at the second line from the top of each group, which corresponds to the $\pi$ component (perpendicular to the magnetic field). The third and fourth lines correspond to the spectra with $45^{\circ}$ and $135^{\circ}$, respectively. These spectra have contributions from both the $\sigma$ component and the $\pi$ component and the contribution depends on the direction of the magnetic field. The spectra with a $45^{\circ}$ linear polarizer (third line) has a larger contribution from $\sigma$ components for groups 7-13, which indicates that the magnetic field is tilted upward. On the other hand, the spectra with a $135^{\circ}\left(=-45^{\circ}\right)$ linear polarizer (fourth line) has a larger contribution from $\sigma$ components for groups 17-20, which indicates that the magnetic field is tilted downward. As seen in Fig. 2, there is an impurity line at pixel number 380 . However, this line is far enough from the $H \alpha$ line to be measured and is not polarized.

Figure 3 shows the spectra of Doppler shifted $H \alpha$ emission for group 7 and group 18 in Fig. 2. The spectra with the $0^{\circ}$ polarizer mainly correspond to the $\sigma$ component, which is polarized parallel to the magnetic field, while the spectra with a $90^{\circ}$ polarizer mainly correspond to the $\pi$ component, which is perpendicular to the magnetic field. The spectra with $45^{\circ}$ and $135^{\circ}$ polarizers are a mixture of the $\sigma$ component and the $\pi$ component and the differences give the polarization angles. The viewing array arranged side by side has slightly different Doppler shifts, while the viewing array arranged up and down has identical Doppler shifts. Therefore the measured Doppler shift for the $0^{\circ}$ and $90^{\circ}$ viewing array is different from that for the $45^{\circ}$ and $135^{\circ}$ viewing array. The differences of the Doppler shifts are corrected for each spectrum in Fig. 3.

The intensity of the $\sigma$ component with a $45^{\circ}$ polarizer is 
larger than that with a $135^{\circ}$ polarizer in the spectra observed at group 7, while the intensity of the $\sigma$ component with a $45^{\circ}$ polarizer is smaller than that with a $135^{\circ}$ polarizer in the spectra observed at group 18. These changes indicate the local pitch angle changes its sign between these two groups. The line broadening of each component is mainly due to the beam divergence. The Doppler shift is determined by the beam velocity, $v$, and intersection angle, $\phi$, between the line of sight and neutral beam line as $v \cos (\phi)$, while the effective Doppler width of line is determined by $v \delta \sin (\phi)$, where $\delta$ is a beam divergence angle. The width of the neutral beam, $W$, is simply expressed as $W=2 \delta L$, where $L$ is the distance from the ion source to the observation point, when $L$ is larger than the focal length of the beam. Then the effective Doppler width of the line is determined by $v(W / 2 L) \sin (\phi)$. The spectra with a $45^{\circ}$ polarizer and $135^{\circ}$ spectra show asymmetry. The asymmetries in the spectra with a $45^{\circ}$ polarizer and a $135^{\circ}$ polarizer are due to a variation of polarization angles and the effective Doppler width due to a finite beam divergence along the line of sight, which will be discussed later. It is noted that the polarization angles are derived from the intensity ratios at each wavelength and not from the line ratio of $\sigma$ and $\pi$ components.

The intensity of $H \alpha$ passing a linear polarizer tilted with respect to the horizontal by $\phi$ can be expressed as $I_{\phi}(\lambda)$ $=(1 / 2) I_{\sigma c}(\lambda)+I_{\sigma l}(\lambda) \cos ^{2}\left(\gamma_{\sigma}(\lambda)-\phi\right)+I_{\pi l}(\lambda) \cos ^{2}\left(\gamma_{\pi}(\lambda)-\phi\right)$, where $I_{\sigma c}(\lambda), I_{\sigma l}(\lambda)$, and $I_{\pi l}(\lambda)$ are the circularly polarized and linearly polarized $\sigma$ component and linearly polarized $\pi$ component, respectively. In general, the polarization angle is given by the intensity ratio of two directions $\left(0^{\circ}\right.$ and $90^{\circ}$ or $45^{\circ}$ and $135^{\circ}$ ) for the linearly polarized $\pi$ component. However, the polarization angle of a circularly polarized $\sigma$ component cannot be determined by the intensity ratio of the two directions. The measurement of the four spectra at $0^{\circ}, 45^{\circ}$, $90^{\circ}$, and $135^{\circ}$ polarization angles enables us to eliminate the circularly polarized component and the overlap of the linearly polarized $\sigma$ component and $\pi$ component and derive the polarization angle both for the $\pi$ component and the $\sigma$ component. Because the $\sigma$ component and the $\pi$ component are perpendicular to each other $\left(\gamma_{\pi}=\gamma_{\sigma}-90\right)$ the polarization angle of the $\sigma$ component $\gamma_{p}\left(=\gamma_{\sigma}\right)$ can be given by $\gamma_{p}(\lambda)$ $=(1 / 2) \tan ^{-1}\left(\left[I_{45}(\lambda)-I_{135}(\lambda)\right] /\left[I_{0}(\lambda)-I_{90}(\lambda)\right]\right)$. Figure 4 shows the polarization angle, $\gamma_{p}$, derived from the $H \alpha$ emission from the neutral beam, which is injected to the plasma and gas target. The polarization angle for the magnetic field determined by the external coils is measured with a gas target. The intensity of $H \alpha$ for the gas target is lower than that from the plasma by one order of magnitude. The polarization angles both for the plasma and the gas target (vacuum magnetic field) show strong wavelength dependence. Since the emission volume moves in the toroidal direction along the line of sight, the polarization angle varies within the beamwidth $W$.

Although there is a significant wavelength dependence in the polarization angle $\gamma_{p}(\lambda)$, the change of the polarization angle (difference from the polarization in the vacuum magnetic field) has almost no wavelength dependence. This is because the poloidal field due to the toroidal current has more or less toroidal symmetry and is constant within the
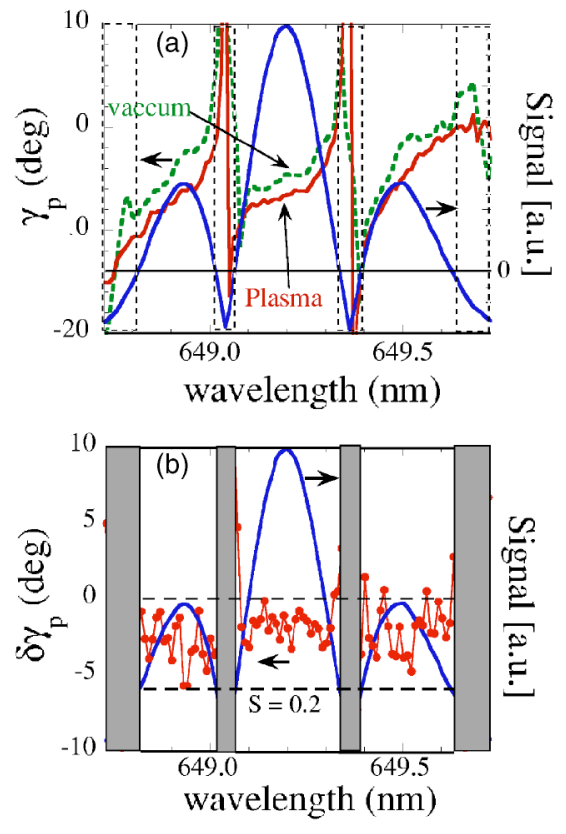

FIG. 4. (Color online) (a) Polarization angle, $\gamma_{p}(\lambda)$, derived from the MSE spectra with and without plasma (vacuum) and (b) shift of polarization angle due to current in the plasma, $\delta \gamma_{p}(\lambda)$, as a function of the wavelength of $H \alpha$. The polarization factor $s=I_{0}(\lambda)-I_{90}(\lambda)$ is also plotted.

beamwidth. The change of the polarization angle is derived by averaging the $\delta \gamma_{p}(\lambda)$ for each component and the error bar is given by the standard deviation of $\delta \gamma_{p}(\lambda)$. The polarization angle has a large uncertainty at the boundary between $\sigma$ and $\pi$ lines, because the polarization factor $\mathrm{s}=I_{0}(\lambda)$ $-I_{90}(\lambda)$ is approaching zero. Therefore, the polarization angles in the region where the polarization factor exceeds 0.2 are used in averaging. The scatter of the change of polarization angle is mainly due to the low signal of the polarization angle in the vacuum magnetic field, not that in the plasma.

\section{EVALUATION OF ROTATIONAL TRANSFORM PROFILE}

In order to derive the rotational transform, the equilibrium code VMEC is used. The radial profile of the polarization angle measured is compared with that calculated with a VMEC database with various pressure profiles and current profiles. The VMEC data consist of a few hundred equilibria with various total pressure, total toroidal current, and current density profiles. Figures 5(a) and 5(b) show the radial profile of the rotational transform in the equilibrium magnetic field which gives the best fit to the polarization angle measured. The total toroidal current measured with a coil is used in this best fit process. Then the central rotational transform depends on the current profile for a given total toroidal current. When the current profile is peaked at the plasma center, the central rotational transform becomes large (small) for the positive (negative) toroidal current. Figure 5(c) shows the differences of polarization angle measured and calculated with various current profiles as a function of central rotational transform. The data clearly show the reduction of the central rotational transform: the rotational transform at $\rho$ $=0.1$ is 0.5 for positive toroidal current and 0.37 for the plasma with negative toroidal current. The best fit rotational 

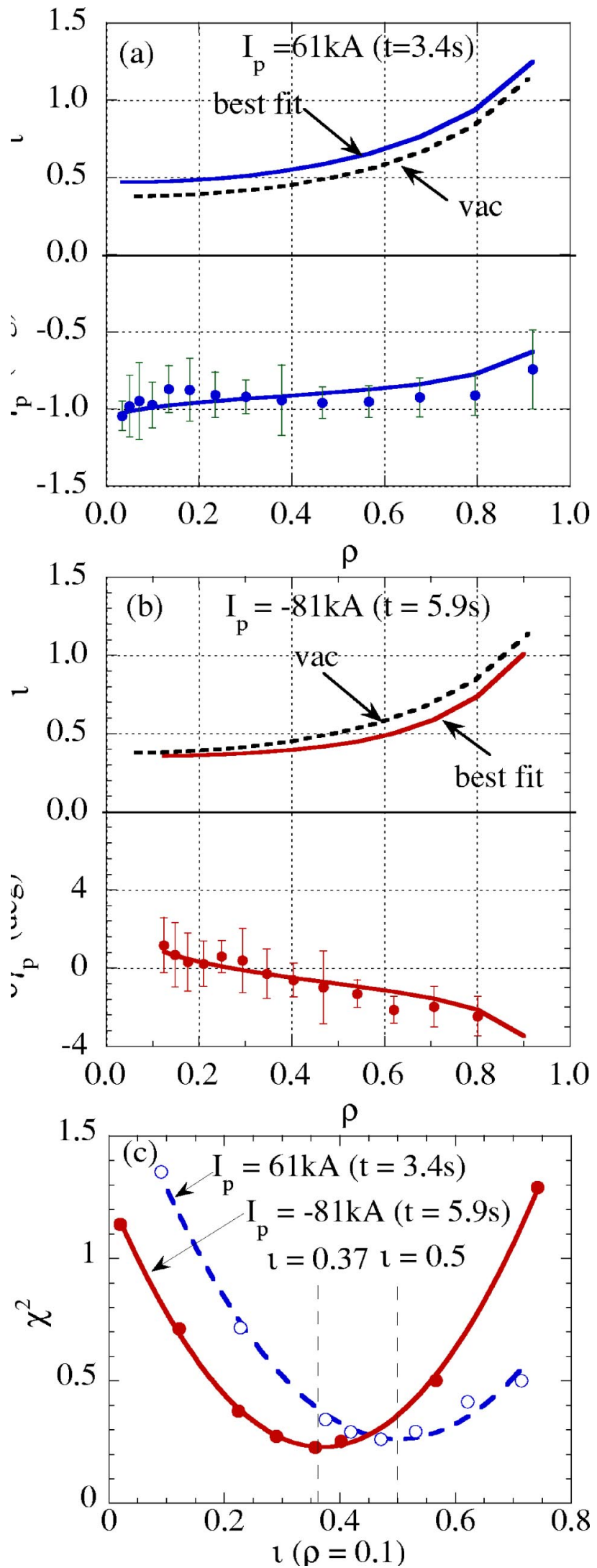

FIG. 5. (Color online) (a) The radial profile of the shift of polarization angle due to plasma and rotational transform for the best fit to the measured polarization angle and vacuum rotational transform in the plasma with (b) a positive toroidal current of $61 \mathrm{kA}$ (co-injection) and (b) a negative toroidal current of $-81 \mathrm{kA}$ (counter-injection) and (c) differences between the shift of polarization angle measured and that calculated for various current profiles as a function of rotational transform at $\rho=0.1$. transform is given by the interpolation between two equilibria near the minimum $\chi^{2}$. The error bar in the polarization angle is mainly due to the low intensity of $H \alpha$ for the gas target. Therefore the scatter of the data is much lower than the error bar. When the direction of the beam driven current switches from the co-direction to the counter-direction, the polarization angle decreases by $1^{\circ}$ near the plasma center at $\rho=0.35$ and by $3^{\circ}$ at $\rho=0.8$, which corresponds to the decrease of the rotational transform from +0.1 to -0.1 , respectively. This measurement shows that the change of the rotational transform near the plasma edge precedes that near the plasma center.

In the plasma with positive toroidal current, the rotational transform in the plasma is larger than that of the vacuum rotational transform by 0.1 . On the other hand, in the plasma with negative toroidal current the rotational transform in the plasma is smaller than that of the vacuum rotational transform near the plasma edge, but the central rotational transform near the plasma center is close to that in the vacuum magnetic field. The difference in the change of rotational transform suggests that the current profile for the plasma with positive toroidal current is more peaked than that for the plasma with negative toroidal current. This is not because of the change of the deposition profile driven by the NBI, but because the current profiles do not reach the steady state phase. It should be noted that both of these data are taken in the plasma in the transient phase. The direction of the NBI is switched from co-injection to counter-injection (co-injection for $0.3-3.3 \mathrm{~s}$ and counter-injection for $3.3-6.3 \mathrm{~s}$ ). The data for the positive current are taken $0.1 \mathrm{~s}$ after the switch-on of the counter-NBI, while the data for the negative current are taken $2.6 \mathrm{~s}$ after the switch-on of the counter-NBI.

\section{DISCUSSION}

The MSE spectroscopy in LHD consists of linear polarizers and a spectrometer. The advantage of this system is that the $H \alpha$ spectrum is always measured. In the MSE system based on the photoelastic modulator, ${ }^{8-10}$ the center of the transmission wavelength of the filter should be tuned to the center of the $H \alpha$ line depending on the Doppler shift, which is determined by the beam energy. In the tokamak, where there is toroidal symmetry of the pitch angle of the magnetic field, the measurements of spectra are not so important because the polarization angle does not depend on the wavelength. However, the measurement of spectra is especially important in LHD, where the pitch angle of the magnetic field changes significantly along the line of sight in a tangential view within the beamwidth, and the polarization angle strongly depends on the wavelength.

Only a few percent difference in the beam energy between the measurements and the calibration in the vacuum magnetic field causes a significant error. For example, the shift of wavelength by $0.1 \mathrm{~nm}$ (3\% of the beam energy) causes a shift of polarization angle by $1-5^{\circ}$. The change of rotational transform $(\delta \iota \sim 0.1)$ due to the noninductive toroidal current driven by the neutral beam, which is $5 \%$ of the 
toroidal current equivalent to the edge poloidal field created by the external helical coils, is measured with MSE spectroscopy in LHD.

\section{ACKNOWLEDGMENTS}

The authors would like to thank the LHD technical staff for their effort to support the experiments in LHD. This work is partly supported by a grant-in-aid for scientific research of MEXT, Japan.
${ }^{1}$ T. Mazzucato et al., Phys. Rev. Lett. 77, 3145 (1996).

${ }^{2}$ C. B. Forest et al., Phys. Rev. Lett. 77, 3141 (1996).

${ }^{3}$ T. Fujita et al., Phys. Rev. Lett. 78, 2377 (1997).

${ }^{4}$ T. Shimozuma et al., Plasma Phys. Controlled Fusion 45, 1183 (2003).

${ }^{5}$ Y. Takeiri et al., Phys. Plasmas 10, 1788 (2003).

${ }^{6}$ K. Ida et al., Phys. Rev. Lett. 91, 085003 (2003).

${ }^{7}$ F. M. Levinton et al., Phys. Rev. Lett. 63, 2060 (1989).

${ }^{8}$ F. M. Levinton et al., Rev. Sci. Instrum. 61, 2914 (1990).

${ }^{9}$ F. M. Levinton et al., Rev. Sci. Instrum. 63, 5157 (1992).

${ }^{10}$ D. Wroblewski et al., Rev. Sci. Instrum. 63, 5140 (1992).

${ }^{11}$ W. Mandle, R. C. Wolf, M. G. von Hellermann, and H. P. Summers, Plasma Phys. Controlled Fusion 35, 1373 (1993).

${ }^{12}$ S. Takayama, K. Ida, S. Okamura, and S. Kado, Rev. Sci. Instrum. 74, 73 (2003). 